\title{
"Small-for-flow" liver failure after extended hepatectomy: a call for update
}

\begin{abstract}
Emond et al. ${ }^{1}$ first described, in 1996, a new syndrome occurring in small grafts in transplantation field, named small for Size Syndrome. After surgery, patients with a small grafts were affected by unpredictable elevation of bilirubin (not linked with surgical procedures), coagulopathy, prolonged cholestasis, portal hypertension and, if severe, ascites. The continuing liver dysfunction predisposes to further complications, including sepsis and gastrointestinal bleedings, leading to a clear liver failure. In the meantime, in liver resection field, the same syndrome was noticed after major hepatectomy ( $>3$ segments resected), and it was named Post-Hepatectomy Liver Failure. With improvement of knowledge, nowadays we know that SFSS and PHLF are two side of the same coin. Different strategies, more in transplantation context, have been described to prevent and to treat the syndrome, but lack of data regarding the resection context is still a matter. In fact, where in transplant, pathophysiological studies and randomized clinical trial have been published to evaluate the etiology and the efficacy of procedures proposed, in resection field we are just translate transplantation experience, without a whole consensus between physician on how to predict, to diagnose, and to treat this post-hepatectomy liver failure. Diagnostic criteria, treatments, and pathophysiology are still unclear, with different opinion between physicians, and also disaccording if small for size and post-hepatectomy liver failures are really the same. Our opinion a part, we think it's time to start to analyze in deep this syndrome that account for more than $60 \%$ of death after major liver resections
\end{abstract}

Keywords: small for size, liver failure, liver surgery, major hepatectomy, small for flow, splenectomy, somatostatin-analouge, transplant, pathophysiological, randomized clinical, coagulopathy, prolonged cholestasis, criteria, treatments; unclear
Volume 3 Issue 2 - 2017

\author{
Simone Famularo, Alessandro Giani, Mauro \\ Montuori, Fabrizio Romano \\ University of Milan-Bicocca Milan, Italy
}

Correspondence: Simone Famularo, School of Medicine and Surgery, University of Milan-Bicocca Milan, Pergolesi 33, 20099 Monza, Italy, Email simone.famularo@gmail.com

Received: April 18, 2017 | Published: May 02, 2017
Abbreviations: SAE, splenic artery embolization; SFFLF, small for flow liver failure; SFSS, small for size syndrome; TLV, total liver volume; FLR, future liver remnant; PVP, portal vein pressure; HVPG, hepatic venous portal gradient

\section{Introduction}

Emond et al. ${ }^{1}$ first described, in $1996,{ }^{1}$ a new syndrome occurring in small grafts in transplantation field, named Small for Size Syndrome. After surgery, patients with a small grafts were affected by unpredictable elevation of bilirubin (not linked with surgical procedures), coagulopathy, prolonged cholestasis, portal hypertension and, if severe, ascites. The continuing liver dysfunction predisposes to further complications, including sepsis and gastrointestinal bleedings, leading to a clear liver failure. In the meantime, in liver resection field, the same syndrome was noticed after major hepatectomy $(>3$ segments resected), and it was named Post-Hepatectomy Liver Failure. With improvement of knowledge, nowadays we know that SFSS and PHLF are two side of the same coin. Different strategies, more in transplantation context, have been described to prevent and to treat the syndrome, but lack of data regarding the resection context is still a matter. In fact, where in transplant, pathophysiological studies and randomized clinical trial have been published to evaluate the etiology and the efficacy of procedures proposed, in resection field we are just translate transplantation experience, without a whole consensus between physician on how to predict, to diagnose, and to treat this post-hepatectomy liver failure. Diagnostic criteria, treatments, and pathophysiology are still unclear, with different opinion between physicians, and also disaccording if small for size and post-hepatectomy liver failures are really the same. Our opinion a part, we think it's time to start to analyze in deep this syndrome that account for more than $60 \%$ of death after major liver resections. ${ }^{2}$

\section{Different opinions, lack of agreement}

Even if imaging shows complete restoration of liver volume after few weeks from surgery, there is no evidence of complete restoration of liver's parenchymal micro architecture when looking at the liver biopsy of same patients taken soon after extended resection or transplantation. ${ }^{3,4}$ In the field of oncological surgery, liver regeneration is a very complex problem when it's approaching a malignancy. In fact, liver volume regeneration is not always accompanied by a quick and complete restoration of liver function. ${ }^{2}$ Often, it has been noticed a distortion in micro and macro architecture of liver cells,${ }^{5}$ with hyperproliferation of hepatocytes, and lack of proper interaction with other types of hepatic cells. Over portal flow and pressure are pointed as the "primum movens" in the pathophysiology of the syndrome. This phenomenon has been studied by Asencio et al. ${ }^{6}$ proposing a new approach to post-hepatectomy liver failure, leading to a predominant role of flow and pressure in the etiophatogenesis, and, consequently, a new management approach for liver surgery and related safe limits.

Because of its small size, liver remnant after major hepatectomy is suddenly submitted to a hyperperfusion induced by the wholemaintained portal vein flow. This seems to cause an adenosine washout in Space of Mall, who leads to hepatic artery vasoconstriction (a phenomenon named Hepatic Arterial Buffer Response, HABR). This acute and relative portal hypertension leads to endothelial damage, ischemic and riperfusion's injuries that explains liver dysfunction.? 
sThis hypothesis came out from living donor liver transplantation's experience, where it has been noticed the good outcome of portal flow and pressure modulation strategies to avoid small-for-size syndrome. In fact, in transplantation context, reducing portal vein pressure (PVP) by portocaval shunt, ${ }^{8}$ or by splenic artery embolization, ${ }^{9}$ or by splenectomy ${ }^{10}$ had been showed to be very effective as prevention treatments. Ogura et al. ${ }^{11}$ strongly showed that LDLT using smallgrafts was feasible provided that Portal Vein Pressure was maintained below $15 \mathrm{mmHg}$. In an oncological context, some experimental studies have showed how low PVP after surgery is a protective factor against histological damage, leading to a better functional regeneration of liver. ${ }^{12-14}$ Recently, Allard MA and colleagues ${ }^{15}$ observed a significantly higher post-hepatectomy PVP in patients who develop post-hepatectomy liver failure (all definitions considered) compared to those who did not. He suggested an intra-operative PVP cut off of $22 \mathrm{mmHg}$, that is associated with $18.4 \%-21.1 \%$ (depending on definitions considered) of likelihood to develop a Small for flow liver failure, and a 90 -days mortality of $12.7 \%$. Several other studies indicate that portal pressure higher than $20 \mathrm{mmHg}$ show a decrease from $85 \%$ to $38 \%$ in their 6-months survival. ${ }^{8}$ Despite this, other authors, recently, have suggested that portal hyperperfusion after extended hepatectomy doesn't induce a hepatic arterial buffer response, but reduces mitochondrial redox state and hepatocellular oxygenation in rat's model. This wouldn't due to a deterioration of microvascular perfusion, but rather due to a relative hyper metabolism of the remnant liver after major resection. ${ }^{16}$

Criteria to diagnose SFFLF are another theme of debate. In 2005, Balzan et $\mathrm{al}^{2}$ have proposed the so-called "50-50 criteria", where an elevation of PT over $50 \%$ of baseline, or INR $>1.7$, and a raise of bilirubin more than $3 \mathrm{mg} / \mathrm{dl}$ on or after 5 th post-operative day, are related to SFFLF development, and to $59 \%$ of mortality after surgery. ${ }^{2}$ The International Study Group of Liver Surgery (ISGLS) has proposed a three-grade classification (Table 1). Another criteria accepted in literature, is the peak of bilirubin $>120 \mu \mathrm{mol} / 1$ after day 5 th. These authors have founded their classifications on their pathophysiological point of view. Thus, this lack of agreement is reflected even in SFFLF definition: according to ISGLS, post-hepatectomy liver failure is "a postoperative acquired deterioration in the ability of the liver to maintain its synthetic, excretory and detoxifying functions, which is characterized by an increased international normalized ratio and concomitant hyperbilirubinemia on or after postoperative day $5 "{ }^{17}$ According to Asencio et al. ${ }^{6}$ who have proposed the new concept of "small-for-flow", SFFLF is "the proportion between the mass of the liver remnant and the blood flow that receives, reflected by the values of portal blood flow and pressure. A disproportionate increase of portal blood flow and portal pressure would lead to sinusoidal injury, ischemic injury due to a compensatory reduction of blood flow in the hepatic artery, and to impairment of liver regeneration". ${ }^{6}$

Table I ISGLS grading system

\section{ISGLS grading system}

Grade A laboratory parameters but requiring no change in the

clinical management of the patient deviation from the regular clinical management but manageable without invasive treatment

Post-hepatectomy liver failure resulting in a

Grade C deviation from the regular clinical management and requiring invasive treatment

\section{Time to clarify}

All these evidences show how we're far away to an objective and whole-accepted model to understand, predict, manage and treat this liver failure in liver resection's field. Even if, in clinical practice, different authors have described different approach to reduce portal flow preventing the syndrome (porto-caval shunt; ${ }^{18}$ splenectomy; splenic artery embolization; ${ }^{19}$ somatostatin-analouge's infusion ${ }^{20}$ ) lack of evidence and correlation permit to have a clear and evidencebased model. Nowadays, size is the center of liver surgery's planning, but, as Asencio have proposed, if portal flow would be definitely demonstrated as the key point, this paradigm could be totally changed.

It would be time to make an effort, and clarify the etiology of this syndrome, to change permanently our approach to liver surgery. In fact, if the evidences related to the portal pressure and flow as "primum movens" would be assessed, our point of view may shift from how much liver's parenchyma we are leaving in patients, to how modulate intra-operatively or post-operatively portal pressure and flow to guarantee the best liver's regeneration after surgery.

\section{Acknowledgements}

None.

\section{Conflict of interest}

Author declares that there is no conflict of interest.

\section{References}

1. Emond JC, Renz JF, Ferrell LD, et al. Functional analysis of grafts from living donors Implications for the treatment of older recipients. Ann Surg. 1996;224(4):544-552.

2. S Balzan, J Belghiti, O Farges, et al. The 50-50 criteria on postoperative day 5: an accurate predictor of liver failure and death after hepatectomy. Ann Surg. 2005;242(6):824-829.

3. Y Panis, McMullan DM, J Emond. Progressive necrosis after hepatectomy and the pathophysiology of liver failure after massive resection. Surgery. 1997;121(2):142-149.

4. R Huang, T Schiano, M Amolat, et al. Hepatocellular proliferation and changes in microarchitecture of right lobe allografts in adult transplant recipients. Liver transpl. 2004;10(12):1461-1467.

5. N Golse, P Bucur, R Adam, et al. New paradigms in post-hepatectomy liver failure. J Gastrointest Surg. 2013;17(3):593-605.

6. J Asencio, J Vaquero, L Olmedilla, et al. Small for flow syndrome: shifting the size paradigm. Medical Hypothesis. 2013;80(5):573-577.

7. P Clavien, C Oberkofler, D Raptis, et al. What is critical for liver surgery and partial liver transplantation: size or quality? Hepatology. 2010;52(2):715-729.

8. O Boillot, B Delafosse, I Mechet, et al. Small-for-size partial liver graft in an adult recipient; a new transplant technique. Lancet. 2002;359(9304):406-407.

9. T Ito, T Kiuchi, H Yamamoto, et al. Changes in portal venous pressure in the early phase after living donor liver transplantation: pathogenesis and clinical implications. Transplantation 2003;75(8):1313-1317.

10. T Hori, Y Ogura, K Ogawa, et al. How transplant surgeons can overcome the inevitable insufficiency of allograft size during adult living donor liver transplantation: strategy for donor safety with a smaller-size graft and excellent recipient results. Clin Transplant. 2012;26(3):E324-E334.

11. Y Ogura, T Hori, El Moghazy W, et al. Portal pressure $<15 \mathrm{mmHg}$ is a key for successful adult living donor liver transplantation utilizing smaller grafts than before. Liver Transpl. 2010;16(6):718-728. 
12. T Asakura, N Ohkohchi, T Orii, et al. Portal vein pressure is the key for successful liver transplantation of an extremely small graft in the pig model. Transpl Int. 2003;16(6):376-382.

13. T Iida, S Yagi, K Taniguchi, et al. Improvement of morphological changes after $70 \%$ hepatectomy with portocaval shunt: preclinical study in porcine model. $J$ Surg Res. 2007;143(2):238-246.

14. T Fukuchi, H Hirose, A Onitsuka, et al. Effects of portal-systemic shunt following $90 \%$ partial hepatectomy in rats. J Surg Res. 2000;89(2):126-131.

15. M Allard, R Adam, P Bucur, et al. Posthepatectomy portal vein pressure predicts liver failure and mortality after major liver resection on noncirrhotic liver. Ann Surg. 2013;258(5):822-829.

16. S Dold, S Richter, O Kollmar, et al. Portal hyperperfusion after extended hepatectomy does not induce a hepatic arterial buffer response (HABR) but impairs mitochondrial redox state and hepatocellular oxygenation. PLoS One. 2015;10(11):e0141877.
17. N Rahbari, O Garden, R Padbury, et al. Posthepatectomy liver failure: a definition and grading by the international study group of liver surgery (ISGL). Surgery. 2011;149(5):713-724.

18. R Troisi, S Ricciardi, P Smeets, et al. Effects of hemiportocaval shunts for inflow modulation on the outcome of small-for-size grafts in living donor liver transplantation. Am J Transplant. 2005;5(6):1397-1404.

19. A Luca, R Miraglia, S Caruso, et al. Effects of splenic artery occlusion on portal pressure in patients with cirrhosis and portal hypertension. Liver Transpl. 2006;12(8):1237-1243.

20. E Matrella, V Valatas, G Notas, et al. Bolus somatostatin but not octreotide reduces hepatic sinusoidal pressure by a NO-independent mechanism in chronic liver disease. Aliment Pharmacol Ther. 2001;15(6):857-864. 